\title{
Monitoring Motor Fluctuations in Patients With Parkinson's Disease Using Wearable Sensors
}

\section{Citation}

Shyamal Patel, Konrad Lorincz, Richard Hughes, Nancy Huggins, John Growdon, David Standaert, Metin Akay, Jennifer Dy, Matt Welsh, and Paolo Bonato. 2009. Monitoring motor fluctuations in patients with Parkinson's disease using wearable sensors. IEEE Transactions on Information Technology in Biomedicine 13(6): 864-873.

\section{Published Version}

doi:10.1109/TITB.2009.2033471

\section{Permanent link}

http://nrs.harvard.edu/urn-3:HUL.InstRepos:4099742

\section{Terms of Use}

This article was downloaded from Harvard University's DASH repository, and is made available under the terms and conditions applicable to Other Posted Material, as set forth at http:// nrs.harvard.edu/urn-3:HUL.InstRepos:dash.current.terms-of-use\#LAA

\section{Share Your Story}

The Harvard community has made this article openly available.

Please share how this access benefits you. Submit a story.

\section{Accessibility}




\title{
Monitoring Motor Fluctuations in Patients With Parkinson's Disease Using Wearable Sensors
}

\author{
Shyamal Patel, Konrad Lorincz, Richard Hughes, Nancy Huggins, John Growdon, David Standaert, \\ Metin Akay, Fellow, IEEE, Jennifer Dy, Matt Welsh, Member, IEEE, and Paolo Bonato, Senior Member, IEEE
}

\begin{abstract}
This paper presents the results of a pilot study to assess the feasibility of using accelerometer data to estimate the severity of symptoms and motor complications in patients with Parkinson's disease. A support vector machine (SVM) classifier was implemented to estimate the severity of tremor, bradykinesia and dyskinesia from accelerometer data features. SVM-based estimates were compared with clinical scores derived via visual inspection of video recordings taken while patients performed a series of standardized motor tasks. The analysis of the video recordings was performed by clinicians trained in the use of scales for the assessment of the severity of Parkinsonian symptoms and motor complications. Results derived from the accelerometer time series were analyzed to assess the effect on the estimation of clinical scores of the duration of the window utilized to derive segments (to eventually compute data features) from the accelerometer data, the use of different SVM kernels and misclassification cost values, and the use of data features derived from different motor tasks. Results were also analyzed to assess which combinations of data features carried enough information to reliably assess the severity of symptoms and motor complications. Combinations of data features were compared taking into consideration the computational cost associated with estimating each data feature on the nodes of a body sensor network and the effect of using such data features on the reliability of SVM-based estimates of the severity of Parkinsonian symptoms and motor complications.
\end{abstract}

Index Terms-Body sensor networks, Parkinson's disease, support vector machines (SVMs), wearable sensors.

Manuscript received February 28, 2009; revised July 24, 2009. First published October 20, 2009; current version published November 4, 2009. This work was supported in part by the National Institute of Neurological Disorders and Stroke under Grant R21NS045401-02, by the National Science Foundation under Grant CNS-0546338, by the Michael J. Fox Foundation for Parkinson's Research, and by gifts from Intel Corporation, Siemens, Microsoft, and Sun Microsystems.

S. Patel and R. Hughes are with the Department of Physical Medicine and Rehabilitation, Harvard Medical School, Spaulding Rehabilitation Hospital, Boston, MA 02114 USA (e-mail: spate119@partners.org; rhughes1@partners.org).

K. Lorincz and M. Welsh are with the Harvard School of Engineering and Applied Sciences, Cambridge, MA 02138 USA (e-mail: konrad@eecs.harvard.edu; mdw@eecs.harvard.edu).

N. Huggins and J. Growdon are with the Massachusetts General Hospital Movement Disorders Unit, Harvard Medical School, Boston, MA 02114 USA (e-mail: huggins.nancy@gmail.com; growdon@helix.mgh.harvard.edu).

D. Standaert is with the University of Alabama at Birmingham, Birmingham, AL 35294 USA (e-mail: dstandaert@uab.edu).

M. Akay is with the School of Biological and Health Systems Engineering, Arizona State University, Tempe, AZ 85287 USA (e-mail: metin.akay@asu.edu).

J. Dy is with the Department of Electrical and Computer Engineering, Northeastern University, Boston, MA 02115 USA (e-mail: jdy.ece@ @mail.com).

P. Bonato is with the Department of Physical Medicine and Rehabilitation, Harvard Medical School, Spaulding Rehabilitation Hospital, Boston, MA 02114 USA and also with the Harvard-MIT Division of Health Sciences and Technology, Cambridge, MA, USA (e-mail: pbonato@ partners.org).

Color versions of one or more of the figures in this paper are available online at http://ieeexplore.ieee.org.

Digital Object Identifier 10.1109/TITB.2009.2033471

\section{INTRODUCTION}

$\mathbf{P}$ ARKINSON's disease affects about $3 \%$ of the population over the age of 65 years and more than 500,000 U.S. residents. The characteristic motor features of the disease include tremor, bradykinesia (i.e., slowness of movement), rigidity (i.e., resistance to externally imposed movements), and impaired postural balance. The primary biochemical abnormality in Parkinson's disease is a deficiency of dopamine due to degeneration of neurons in the substantia nigra pars compacta. Current therapy is based on augmentation or replacement of dopamine, using the biosynthetic precursor levodopa or drugs that activate dopamine receptors [1]. These therapies are successful for some time, but most patients eventually develop motor complications [2]. Complications include wearing-off, the abrupt loss of efficacy at the end of each dosing interval, and dyskinesias, involuntary and, at times, violent writhing movements. Wearing-off and dyskinesias produce substantial disability, and frequently interfere with medical therapies [3], [4]. Furthermore, variations in the severity of symptoms and motor complications (referred to as "motor fluctuations") are observed during dosing intervals.

Currently available tools for monitoring motor fluctuations are limited [5], [6]. In clinical practice, information about motor fluctuations is usually obtained by asking patients to recall the number of hours of ON (i.e., when medications effectively attenuate tremor) and OFF time (i.e., when medications are not effective). This kind of self-report is subject to perceptual bias (e.g., patients often have difficulty distinguishing dyskinesia from other symptoms) and recall bias. Another approach is the use of patient diaries, which can improve reliability by recording symptoms as they occur, but does not capture many of the features useful in clinical decision making [7].

Researchers have investigated for some time the use of sensing technology to monitor Parkinsonian symptoms in the home and community settings. Ghika et al. [8] and Spieker et al. [9] were among the first investigators to explore the use of accelerometers and other sensing technology to monitor patients over extended periods of time. Until recently, however, the technology was not adequate to clinically apply these methods. Recent advances in miniature sensor technology, wireless communication, signal processing, and pattern recognition have dramatically changed this situation. Consequently, our research group [10], [11] and others [12]-[16] have focused their efforts on leveraging such advances to develop systems capable of monitoring longitudinal changes in the severity of Parkinsonian symptoms and motor complications. Such systems could facilitate the titration of medications in patients with late stage Parkinson's disease showing severe motor fluctuations. 
Despite recent advances in wearable technology [17] and the development of software that optimizes the use of resources in body sensor networks [18], [19], the use of low-power radios based on the IEEE 802.15.4 standard that has been adopted for implementing the majority of the available body sensor networks is still marked by excessive power consumption thus preventing long-term monitoring of patients unless one implements ad hoc strategies. To decrease power consumption requirements, our research team and others have pursued solutions that minimize the amount of information to be transferred wirelessly from the nodes to the base station of the monitoring system. This goal can be met by extracting (on the body sensor network nodes) and wirelessly transmitting features estimated from the raw data, instead of transmitting the raw data itself.

A limitation of previous work toward developing systems to monitor patients with Parkinson's disease is the lack of integration between wearable technology and the algorithms proposed to estimate the severity of Parkinsonian symptoms and motor complications. Previous work has not carefully considered the need for designing signal processing procedures that can be implemented on the nodes of a body sensor network thus minimizing the amount of data to be transferred from the network nodes to the base station. The use of data features that can be estimated on the network nodes and its impact on the error affecting the estimates of the severity of symptoms and motor complications (derived via pattern recognition techniques implemented on a computer connected with the body sensor network) has never been assessed before. Another limitation of previous studies is their narrow focus on a single symptom (e.g., tremor) or a single motor complication (e.g., dyskinesia). Clinical management of patients with Parkinson's disease requires instead that complex interactions of medications with multiple symptoms and motor complications be monitored over time, assessed, and integrated into algorithms for the clinical management of these patients.

\section{MATERIALS AND METHODS}

\section{A. Data Collection}

Twelve individuals were recruited in the study, ranging in age from 46 to 75 years, with a diagnosis of idiopathic Parkinson's disease (Hoehn \& Yahr stage 2 to 3, i.e., mild to moderate bilateral disease) [20]. Subjects delayed their first medication intake in the morning so that they could be tested in a "practically defined OFF" state (baseline trial). This approach is used clinically to observe patients during their most severe motor symptoms. Subjects were instructed to perform a series of standardized motor tasks utilized clinically to evaluate patients with Parkinson's disease. These motor tasks are part of the motor section of the Unified Parkinson's Disease Rating Scale [21] and included finger-to-nose (reaching and touching a target), finger tapping, repeated hand movements (opening and closing both hands), heel tapping, quiet sitting, and alternating hand movements (repeated pronation/supination movements of the forearms). Fig. 1 shows pictures taken during the execution of these tasks. After completion of the baseline trial, subjects took their medications and were then tested using the same procedure every $30 \mathrm{~min}$. Data was collected during seven trials performed at intervals of $30 \mathrm{~min}$. Video recordings were made during each trial so that a clinical evaluation of the severity of tremor, bradykinesia, and dyskinesia could be performed later on. A clinical expert examined the video recordings and provided clinical scores representing the severity of tremor, dyskinesia, and bradykinesia for each motor task performed by patients during each trial. Such assessment relied upon the Unified Parkinson's Disease Rating Scale [20], which provides five discrete levels of severity ranging from 0 to 4 . Clinical scores provided by clinicians via visual examination of the video recordings were compared with estimates derived from the accelerometer data using the methods described in the following sections.

Uniaxial accelerometer sensors positioned on the upper and lower limbs (as shown in Fig. 2) were used to gather movement data during performance of the earlier-described standardized series of motor tasks. The axes of the accelerometers utilized to capture movements of the upper extremities were oriented in the anteroposterior direction (for subjects standing with arms on the side of the body and palms touching the thighs). The axes of the accelerometers positioned on the lower extremities were oriented distally ("down" when subjects were standing). In this pilot study, we did not use wireless sensors because a suitable platform was not available when the study was started. Rather, we gathered sensor data via a tethered ambulatory system (Vitaport 3, Temec BV, The Netherlands). Accelerometers with the same characteristics as the ones utilized as part of the wireless sensor platform developed later on (as described next) were used for the experiments. Data was sampled at $100 \mathrm{~Hz}$.

\section{B. Feature Extraction}

Raw accelerometer data were high-pass filtered with a cutoff frequency of $1 \mathrm{~Hz}$ to remove the effect of gross changes in the orientation of body segments [13]. An additional filter was applied to isolate the frequency components of interest for estimating each symptom or motor complication. Specifically, the time series were bandpass filtered with bandwidth $3-8 \mathrm{~Hz}$ for the analysis of tremor, and low-pass filtered with a cutoff frequency of $3 \mathrm{~Hz}$ for the analysis of bradykinesia and dyskinesia. All filters were implemented as infinite impulse response (IIR) filters based on an elliptic design.

The accelerometer time series were segmented using a rectangular window randomly positioned throughout the recordings gathered during performance of each motor task [10]. Features were extracted from 30 data segments (i.e., epochs) for each motor task for each trial. Five different types of features were estimated from the accelerometer data: the range of amplitude of each channel, the root mean square (rms) value of each accelerometer signal, two cross-correlation-based features (i.e., the peak of the normalized cross-correlation function derived from pairs of accelerometer time series and the time lag corresponding to such peak value; the normalization of the cross-correlation function limited its values between -1 and 1), two frequency-based features (i.e., the dominant frequency component and the ratio of the energy associated with the dominant frequency component to the total energy), and the signal entropy [22]. The choice of such 


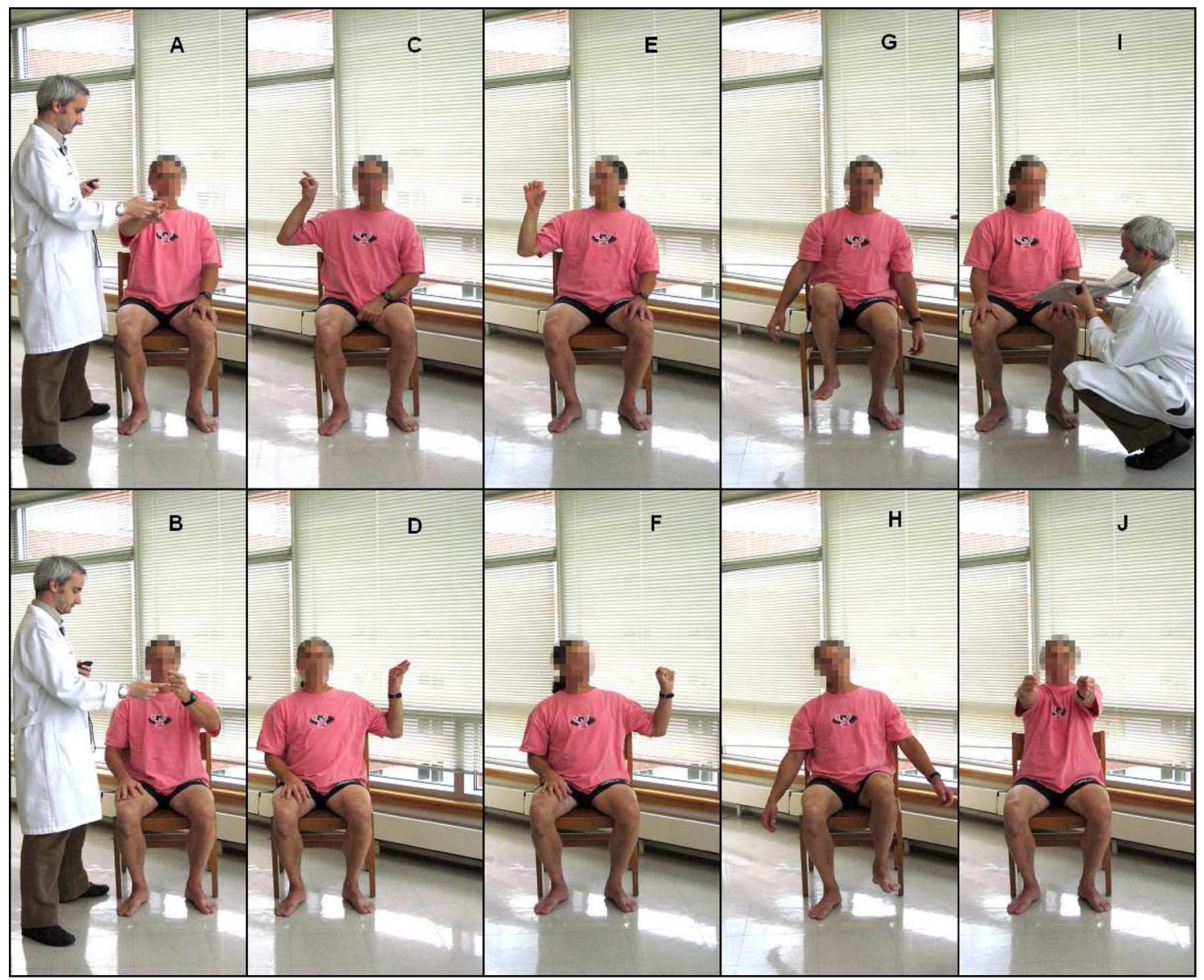

Fig. 1. Motor tasks performed by subjects during each trial. The tasks included: (a) and (b) finger to nose (right and left), (c) and (d) finger tapping, (e) and (f) opening/closing the hands, (g) and (h) heel tapping, (i) sitting, and (j) alternating hand movements.

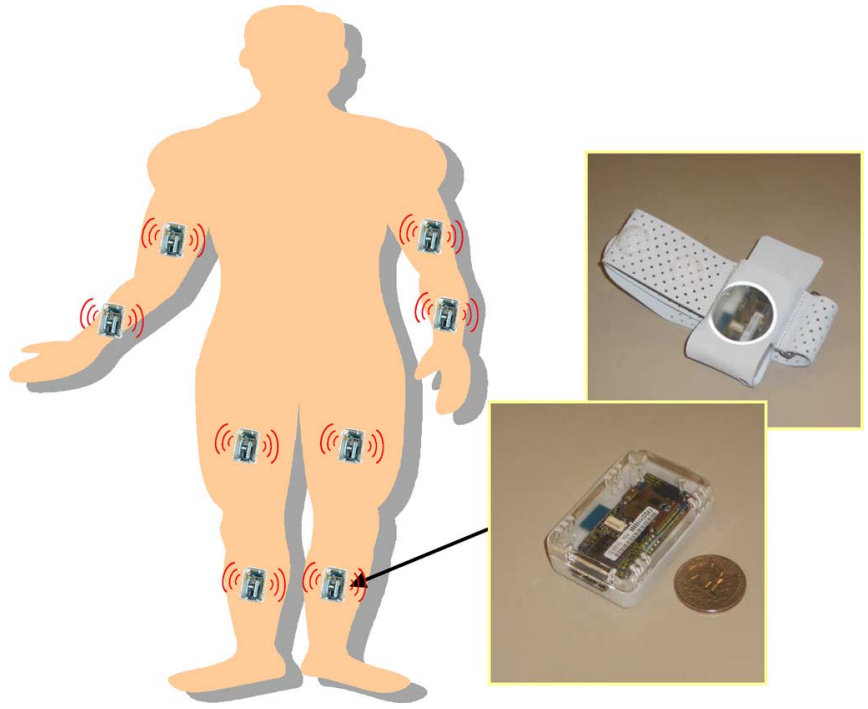

Fig. 2. Schematic representation of the position of sensors on the body to gather accelerometer data. The SHIMMER platform we envision to use in future studies is also shown.

features was based on previous work that allowed us to identify features sensitive to changes in the severity of Parkinsonian symptoms and motor complications and motor tasks that are suitable to capture movement characteristics associated with
TABLE I

MOTOR TASKS UTILIZED FOR ESTIMATION OF SEVERITY SCORES

\begin{tabular}{l|c|c|c|}
\hline Motor Task & Tremor & Bradykinesia & Dyskinesia \\
\hline Finger to Nose & $\checkmark$ & $\checkmark$ & $\checkmark$ \\
\hline Finger Tapping & $\checkmark$ & & $\checkmark$ \\
\hline $\begin{array}{l}\text { Open/Close Hands } \\
\text { Heel Tapping }\end{array}$ & $\checkmark$ & & \\
\cline { 2 - 4 } $\begin{array}{l}\text { Sitting } \\
\text { Alt. Hand Movements }\end{array}$ & $\checkmark$ & $\checkmark$ & \\
\cline { 2 - 4 } & $\checkmark$ & & $\checkmark$ \\
\hline
\end{tabular}

tremor, bradykinesia, and dyskinesia [10]. Table I provides a list of the motor tasks used to assess the severity of tremor, bradykinesia, and dyskinesia. It is worth mentioning that, while in some cases, the motor tasks themselves were inadequate to estimate the severity of a given symptom or motor complication (e.g., the heel tapping task does not lend itself to classifying the severity of dyskinesia), in other cases, the decision to exclude a motor task was made based on the lack of appropriate sensors. For instance, the open/close hands task could potentially be suitable to estimate the severity of bradykinesia, but the setup would require the use of either inertial sensors on the hands or the use of electromyography to capture the activity of muscles controlling the rhythmic opening and closing of the hands. 


\section{Support Vector Machines (SVMs)}

Estimating accelerometer data features for all the sensors utilized in the experiments produced large feature sets. In pilot work [10], [11], projection algorithms, clustering techniques, and measures of cluster quality were used to assess the suitability of the accelerometer-based feature sets to estimate the severity of tremor, bradykinesia, and dyskinesia. Encouraged by the results of our pilot work, we decided to develop a classifier to estimate the severity of Parkinsonian symptoms and motor complications based on accelerometer data features. We chose to use SVMs [23] due to the success of this approach in many classification problems. SVMs demonstrate good generalization performance [23]. We used the PRTools4 toolbox to implement SVM [24]. The specific SVM implementation we adopted relies on the one-versus-rest approach to tackle the multiclass classification problem. Three different kernels (i.e., exponential, radial basis, and polynomial kernels) were utilized and results were compared.

\section{Optimization of the Algorithms}

Optimization of the algorithms was achieved by minimizing the error affecting the estimates of clinical scores measuring the severity of tremor, bradykinesia, and dyskinesia that we derived using SVM. Values derived using the earlier-described methods were compared with the scores provided by clinicians based on visual inspection of video recordings taken while patients performed a standardized series of motor tasks. For all the procedures described next, we used a tenfold cross-validation technique. The technique was utilized to analyze data on an individual basis, i.e., the algorithms were trained on a subjectby-subject basis. This approach is justified by the fact that our interest is for developing methods to track longitudinal changes in the severity of Parkinsonian symptoms and motor complications on an individual basis rather than on a group basis.

First, we studied the effect of the length of the window used to select epochs of accelerometer data and derive data features. Our objective was to achieve average estimation errors below $5 \%$. This value conservatively approximates the interrater variability that marks the use of the Unified Parkinson's Disease Rating Scale, the clinical scale utilized in this study to assess the severity of symptoms and motor complications [21]. We utilized window lengths ranging from 1 to $7 \mathrm{~s}$ with increments of $1 \mathrm{~s}$. Then, we explored the effect of three different SVM kernels: polynomial, exponential, and radial basis kernels. We tested four different values $(0.1,10,100$, and 1000) of the misclassification cost parameter "C" used to train the SVM. Results were also analyzed to compare the error affecting the estimates of the severity of tremor, bradykinesia, and dyskinesia calculated by using feature sets from data recorded during performance of different motor tasks. These analyses aimed at identifying motor tasks suitable to achieve reliable estimates of the severity of Parkinsonian symptoms or motor complications. Finally, we assessed the impact of individual data features and combinations of data features on the error affecting the estimates provided by SVM. We considered five feature types: data range, rms value, cross-correlation-based features, frequency-based features, and signal entropy. Analyses were performed for each feature type (i.e., feeding the SVM with only one feature type) and all possible combinations of features (i.e., all possible combinations of two feature types, three feature types, four feature types, and all five feature types). Consequently, a total of 31 different combinations of feature types were tested (i.e., $\left.\sum_{r=1}^{5} \frac{n !}{r !(n-r) !}\right|_{n=5}$ ).

\section{E. Toward the Use of a Body Sensor Network}

In this study, data were collected using a tethered ambulatory system in the laboratory environment. For clinical applications in the home and community settings, it would be desirable to utilize a wireless system. The sensor platform we envision to use for clinical application of the methodologies herein investigated is the Intel Digital Health Group's Sensing Health with Intelligence, Modularity, Mobility, and Experimental Reusability (SHIMMER) [25].

SHIMMER (Fig. 2) is a body sensor network that consists of nodes equipped with a TI MSP430F1611 microprocessor, a Chipcon CC2420 IEEE 802.15.42.4 GHz radio, a MicroSD card slot, a triaxial microelectromechanical systems accelerometerthe Freescale MMA7260Q — and, optionally, a Bluetooth radio that allows streaming of sensor data at high rates. The SHIMMER device measures $1.75^{\prime \prime} \times 0.8^{\prime \prime} \times 0.5^{\prime \prime}$ and weighs $10 \mathrm{~g}$. The MicroSD card slot allows up to 2 GB of flash memory. Given the amount of flash memory available on the body sensor network nodes in the application discussed in this paper, wireless communication can be used solely when the protocol requires synchronizing multiple units, and when researchers are interested in performing spot checks on the quality of the data. This characteristic of the SHIMMER platform substantially reduces power consumption compared to the alternative in which no data are stored on the nodes and the radio is constantly utilized.

To monitor the quality of the recordings, one can rely upon estimating data features on the nodes with available computational resources and wirelessly transmitting only such data features. Building this capability into a body sensor network would allow clinical personnel to check that data captured during the monitoring interval are satisfactory and carry the information they need. One challenge to implementing this strategy is the limited availability of computational resources on the nodes. This requires performing a tradeoff between the relevance of the clinical information captured by a given data feature and the computational cost associated with its estimation. Also, one has to consider that estimating certain data features requires the use of the radio to transmit data from one node to another This is the case for the cross-correlation-based features. Features should therefore be ranked first according to the "cost" associated with their estimation (including computational cost and power consumption associated with data transmission) and then sorted according to their impact on the error affecting the estimates of the severity of Parkinsonian symptoms and motor complications.

To assess the computational cost of deriving each of the data features of interest on the SHIMMER platform, we estimated 


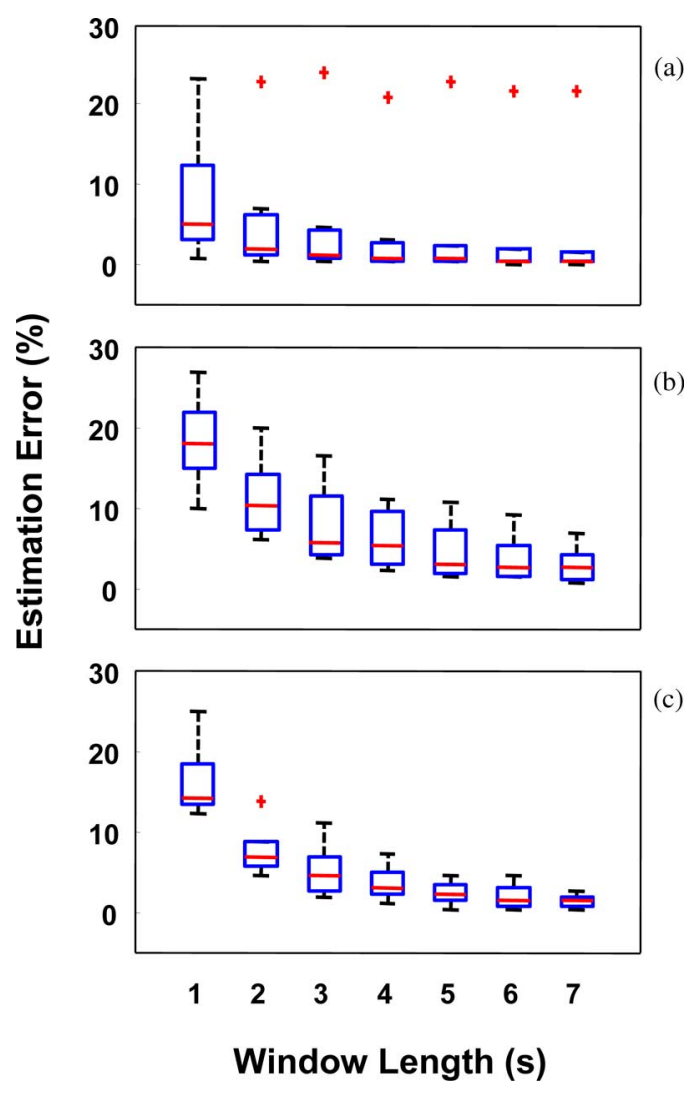

Fig. 3. Effect of the window length on the error affecting the estimates of clinical scores derived via analysis of the accelerometer data. Estimation error values were averaged across all the motor tasks to derive the box plots for (a) tremor, (b) bradykinesia, and (c) dyskinesia. Results were derived on a subjectby-subject basis and are shown as aggregate data.

the time needed to compute such features for a $5 \mathrm{~s}$ epoch of accelerometer data. For this purpose, we implemented and executed procedures to calculate data features on the sensor nodes (i.e., on the MSP430F1611 processor). Start and end times of the interval required for the data feature estimation were measured by reading the value of a register incremented using a $32 \mathrm{kHz}$ clock. Estimates were obtained for 50 epochs and average values of the time needed to compute different features were compared.

\section{RESULTS}

\section{A. Selecting the Optimal Window Length}

Fig. 3 shows the results achieved when segmenting accelerometer data with different window lengths. In the box plot, the horizontal line represents the median value, the box shows the interval from the lower quartile to the upper quartile, and the whiskers show the range covered by the data (excluding outliers). Outliers, indicated each by a plus sign, were detected as points exceeding 1.5 times the interquartile range. Estimation error values were obtained by utilizing all feature types and by implementing SVM using a third-order polynomial kernel. Similar results were obtained using other kernels. A significant decrease in the average estimation error for tremor, bradykinesia, and dyskinesia can be seen when increasing the window length from 1 to $4 \mathrm{~s}$. Error values appear to plateau with a window of $5 \mathrm{~s}$ or longer. The median values of the estimation errors for a window of $5 \mathrm{~s}$ were below the $5 \%$ target. The longer the windows we adopt, the larger the collected dataset would need to be in order to avoid high correlation among epochs utilized to derive each feature value. Therefore, one should adopt the shorter window that allows one to achieve the desired estimation error. The results summarized in Fig. 3 supported the choice of a $5 \mathrm{~s}$ window for extracting data features to estimate the severity of Parkinsonian symptoms and motor complications.

Interestingly, the pattern of decrease in estimation error values observed when increasing the window length utilized to segment the accelerometer data into epochs is slightly different for tremor compared to bradykinesia and dyskinesia. A shorter window appears to be suitable for estimating clinical scores of tremor compared to the window needed to reliably estimate the severity of bradykinesia and dyskinesia. This observation is not unexpected. In fact, tremor is marked by a rhythmic component of movement between 4 and $7 \mathrm{~Hz}$. A relatively short window therefore captures this behavior reliably. Also, the presence of several outliers is shown in Fig. 3 for estimating tremor (more than bradykinesia and dyskinesia). A detailed inspection of the data indicated that the outliers for tremor were due to the results for one individual whose recordings appeared to be marked by low SNR. This observation suggests that the proposed method performs well for patients with severe tremor and that performance of the algorithms worsens for patients with mild to moderate tremor (therefore showing smaller displacement of the body segments with lower SNRs marking the recordings). The limited number of patients tested in this study did not allow us to perform additional analyses to explore the correlation between the reliability of the results and the severity of tremor. For bradykinesia and dyskinesia, we anticipated the need for using a longer window to segment the data than the one used for tremor because bradykinesia and dyskinesia are marked by lower frequency components than tremor. Bradykinesia by definition consists of slower, lower frequency movements. Dyskinesia is marked by "bursts" of movements that can be observed every few seconds. It is not surprising, therefore, that the estimation error values we derived for a window length of 1 and $2 \mathrm{~s}$ were higher for bradykinesia and dyskinesia compared to tremor.

\section{B. Selecting the Optimal SVM Kernel}

Fig. 4 shows a box plot of the results obtained when different kernels were utilized to implement the SVM. Estimation error values were derived by averaging results for all motor tasks for each Parkinsonian symptom and motor complication. Results were derived on a subject-by-subject basis and shown as aggregate data. Although results were derived for all window length values, Fig. 4 shows only the results obtained when a $5 \mathrm{~s}$ window was used to segment the accelerometer data. No major differences were observed in estimation error values across the three kernels. Interestingly, differences were noted when the misclassification cost parameter " $\mathrm{C}$ " that led to minimum estimation 


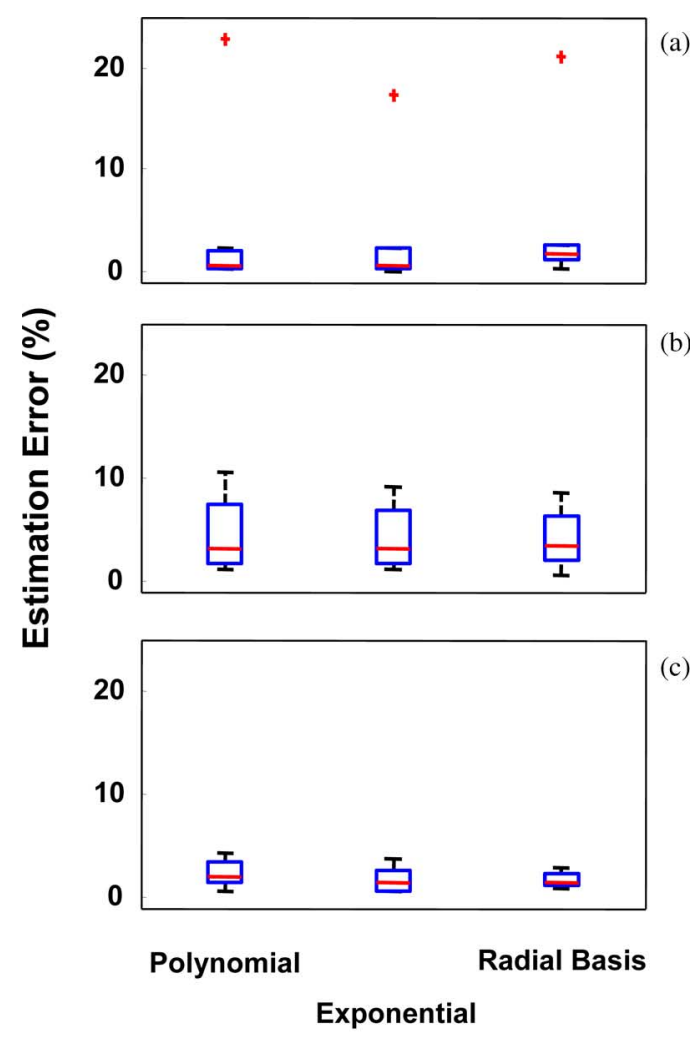

Kernel Type

Fig. 4. Effect of different SVM kernels on the error affecting the estimates of clinical scores derived via analysis of the accelerometer data. Estimation error values were averaged across all the motor tasks to derive the box plots for (a) tremor, (b) bradykinesia, and (c) dyskinesia. Results were derived on a subject-by-subject basis and are shown as aggregate data.

error values for each of the kernels was taken into consideration. In general, a decrease in error values was observed for all kernels when we increased the misclassification cost parameter " $C$ " from 0.1 to 1000 . This result was expected because the complexity of the classifier increases with increasing "C" values. No significant decrease in error value was observed for the results derived using the polynomial kernel for " $\mathrm{C}$ " values greater than 10 , whereas a significant decrease in estimation error value for the exponential and the radial basis kernels was observed when the "C" value was further increased to 100 . This observation suggests that the polynomial kernel discriminates the classes better than the other two kernels.

\section{Selecting Suitable Motor Tasks}

Results of the analyses that we performed to identify motor tasks suitable to estimate the severity of each symptom and motor complication are summarized in Fig. 5. Data features for these results were estimated using a $5 \mathrm{~s}$ window. The SVM was built using a third-order polynomial kernel. Results were derived on a subject-by-subject basis and shown as aggregate data via box plots for each symptom and motor complication for the motor tasks that we selected for the study, as shown in

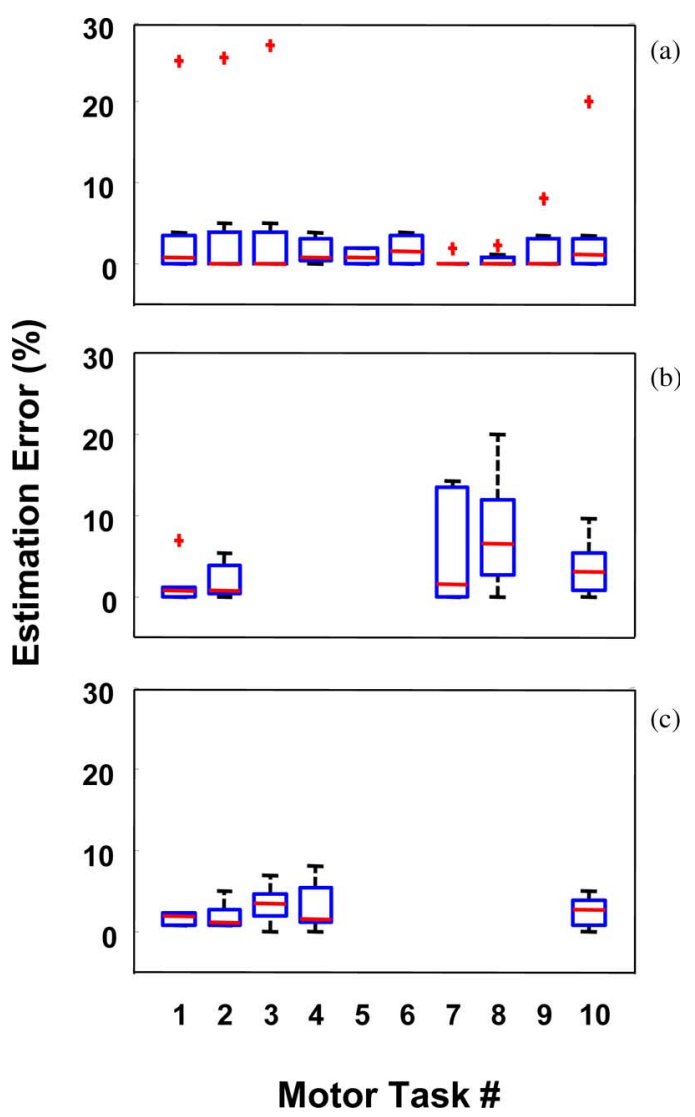

Fig. 5. Effect of selecting different motor tasks on the error affecting the estimates of clinical scores derived via analysis of the accelerometer data. Results are shown for (a) tremor, (b) bradykinesia, and (c) dyskinesia. Box plots of estimation errors are shown for the following motor tasks: Task\#1) finger to nose-right; Task\#2) finger to nose-left; Task\#3) finger tappingright; Task\#4) finger tapping-left; Task\#5) open/close hand-right; Task\#6) open/close hand-left; Task\#7) heel tapping-right; Task\#8) heel tappingleft; Task\#9) sitting; and Task\#10) alternating hand movements. Results were derived on a subject-by-subject basis and are shown as aggregate data.

Table I. Results are not shown for motor tasks not suitable to build a classifier of a given symptom or motor complication.

In general, no major differences were observed in the estimation error values obtained by using feature sets pertaining to different motor tasks, although the use of data from certain tasks appeared to achieve lower estimation error values and variability of the results compared to other tasks. The fact that several tasks appeared to be suitable for estimating the severity of each symptom and motor complication is an important result. This observation suggests that Parkinsonian symptoms and motor complications lead to distinct features of movement that can be captured irrespective of the specific motor task a patient is engaged into. This is a very promising result because it indicates that there is high likelihood that the analyses presented in this manuscript could be extended to motor tasks associated with activities of daily living.

\section{Selecting Accelerometer Features}

Table II summarizes the results derived to assess the effect of individual data feature types and combinations of feature types 
TABLE II

EFFECT OF UTILIZING DifFERENT NUMBERS OF FEATURE TYPES

\begin{tabular}{|c|c|c|c|c|c|}
\hline & $\begin{array}{l}1 \text { Feature } \\
\text { Type }\end{array}$ & $\begin{array}{l}2 \text { Feature } \\
\text { Types }\end{array}$ & $\begin{array}{c}3 \text { Feature } \\
\text { Types }\end{array}$ & $\begin{array}{c}4 \text { Feature } \\
\text { Types }\end{array}$ & $\begin{array}{c}5 \text { Feature } \\
\text { Types }\end{array}$ \\
\hline 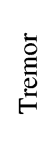 & $\begin{array}{c}6.6( \pm 8.0) \\
22.4( \pm 36.4)\end{array}$ & $\begin{array}{c}3.1( \pm 2.9) \\
8.2( \pm 12.5)\end{array}$ & $\begin{array}{l}2.5( \pm 3.3) \\
3.9( \pm 4.0)\end{array}$ & $\begin{array}{l}2.7( \pm 3.3) \\
3.4( \pm 4.6)\end{array}$ & $\begin{array}{c}2.8 \\
( \pm 3.6)\end{array}$ \\
\hline 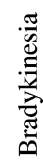 & $\begin{array}{c}2.2( \pm 3.3) \\
10.1( \pm 6.5)\end{array}$ & $\begin{array}{l}1.6( \pm 2.7) \\
7.3( \pm 8.0)\end{array}$ & $\begin{array}{l}1.4( \pm 2.2) \\
3.4( \pm 4.1)\end{array}$ & $\begin{array}{l}1.4( \pm 3.0) \\
1.7( \pm 2.9)\end{array}$ & $\begin{array}{c}1.7 \\
( \pm 3.7)\end{array}$ \\
\hline 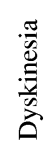 & $\begin{array}{c}3.7( \pm 3.0) \\
25.5( \pm 13.6)\end{array}$ & $1.9( \pm 2.7)$ & $\begin{array}{c}1.8( \pm 1.8) \\
7.4( \pm 11.2)\end{array}$ & $\begin{array}{l}1.6( \pm 2.1) \\
2.7( \pm 2.7)\end{array}$ & $\begin{array}{c}1.2 \\
( \pm 0.9)\end{array}$ \\
\hline
\end{tabular}

The best and worst results (percent estimation error) are shown for each number of feature types, with the exception of the case in which all five feature types were considered. Mean and standard

deviation of the percentage estimation error were derived by pulling together data for all the subjects.

on the reliability of the estimates derived for clinical scores of tremor, bradykinesia, and dyskinesia. Error values were computed for estimates obtained using a window of $5 \mathrm{~s}$ to derive epochs for the computation of data features and applying SVM with a third-order polynomial kernel. Results are shown for a single feature type, two feature types, etc., up to all five feature types. Numerical values shown in Table II are for the minimum and maximum estimation error values for each symptom and motor complication. Average and standard deviation values across subjects were derived for the best (i.e., minimum estimation error value) and worst (i.e., maximum estimation error value) cases.

Results for tremor show that the estimation error value can be as low as $6.6 \%$ even when using a single feature type. This result was obtained using the signal entropy feature. When excluding the signal entropy feature and cross-correlation-based features (the two feature types that were found to require the larger amount of body sensor network resources-see results below), the smallest estimation error value was obtained by relying on frequency-based features and was equal to $12.9 \%$. A decrease in estimation error value was achieved when combinations of two and three feature types were utilized. With two feature types, the lowest average error $(3.1 \%)$ was obtained by utilizing rms value and data range features. When three feature types were utilized, the lowest average estimation error $(2.5 \%)$ was achieved by using the rms value, the data range, and the signal entropy features. A slightly higher estimation error (3.4\%) was achieved using rms value, data range, and frequency-based features.

Results for bradykinesia showed that, in general, clinical scores for this symptom can be estimated with higher reliability (i.e., lower estimation error) than scores for tremor. When using only one feature type, an estimation error value as low as $2.2 \%$ was achieved by utilizing cross-correlation-based features. The lowest value of estimation error that was achieved without using cross-correlation-based features and the signal entropy feature was $6.1 \%$. This value of estimation error was obtained by using frequency-based features. When combinations of two feature types were considered, the lowest estimation error achievable without using cross-correlation-based features and the signal entropy feature was $2.5 \%$. This value of estimation error was obtained combining data range and frequency-based features. When three feature types were used, the best result was achieved by combining rms value, cross-correlation-based, and signal entropy feature types. The combination not using crosscorrelation-based and signal entropy feature types was marked by an estimation error of $2.2 \%$.

Results for dyskinesia showed estimation error values similar to those obtained for bradykinesia. An estimation error value of $3.7 \%$ was achieved using the signal entropy feature. The minimum estimation error value achieved without relying on cross-correlation-based and signal entropy feature types was obtained by relying on the rms value feature and was equal to $6.0 \%$. When using two feature types, an estimation error value of $1.9 \%$ was obtained by using cross-correlation-based features and the signal entropy feature. The best result achieved when combining features that did not include cross-correlation-based features and the signal entropy feature was the one derived by combining the rms value and the range of amplitude of the accelerometer time series, in which case the estimation error was equal to $4.9 \%$. The best result for combinations of three feature types was an estimation error of $1.8 \%$ (achieved when using frequency-based, cross-correlation-based, and signal entropy feature types). A slightly increased estimation error (i.e., 3.2\%) was obtained when using a combination of feature types not including cross-correlation-based features and the signal entropy feature.

\section{E. Estimating Signal Features on the Nodes of a Body Sensor Network}

Table III summarizes the time that we estimated to be necessary to compute each data feature over a $5 \mathrm{~s}$ window for data sampled at $100 \mathrm{~Hz}$ on the nodes of the body sensor network. Estimating data range feature values required approximately $2.5 \mathrm{~ms}$. The estimation of the rms feature value required about $20 \mathrm{~ms}$. Performing a fast Fourier transformation (FFT) required about $450 \mathrm{~ms}$. Its computation accounted for the majority of the time needed to estimate the dominant frequency component ( $489 \mathrm{~ms}$ ) and the ratio of energy associated with the dominant frequency component to the total energy (508 ms). Estimating the signal entropy value required about $1.1 \mathrm{~s}$. Estimating cross-correlation-based features required more than $1.6 \mathrm{~s}$. These results suggest that the data range and $\mathrm{rms}$ value features can be estimated on the nodes of the body sensor network without major interference with other operations (e.g., data sampling and transferring data to the MicroSD card), and that estimating features requiring to perform an FFT is also feasible on the nodes of the body sensor network but at a greater 
TABLE III

ESTIMATES OF THE TIME REQUiRED to CALCULATE FEATURES

\begin{tabular}{l|c}
\hline Feature & Computational Time (ms) \\
\hline Data Range & 2.5 \\
\cline { 2 - 2 } Root Mean Square Value & 20 \\
\cline { 2 - 2 } Dominant Frequency & 489 \\
\cline { 2 - 2 } Ratio of Energy & 508 \\
\cline { 2 - 2 } Signal Entropy & 1100 \\
\cline { 2 - 2 } Peak Cross-Correlation & 1640 \\
\cline { 2 - 2 } & 1640 \\
\hline
\end{tabular}

computational cost. Furthermore, the results suggest that the signal entropy feature should only be used for off-line analysis of the data as its estimation requires more than $1 \mathrm{~s}$ on the node. Our results also indicate that cross-correlation-based features should be only estimated off-line. Besides, it must be observed that estimation of cross-correlation-based features on the body sensor nodes would require transmitting at least one of the accelerometer time series between nodes, leading to significant power consumption.

\section{DisCUSSION AND CONCLUSION}

In this study, we proposed a new method to estimate the severity of tremor, bradykinesia, and dyskinesia from accelerometer data and performed a thorough assessment of several parameters of the proposed algorithms. It was determined that a window of $5 \mathrm{~s}$ (utilized to derive data segments and extract features from the accelerometer time series) is optimal to achieve minimum estimation error, while allowing the utilization of recordings of only $30 \mathrm{~s}$. SVM were implemented and the use of three different kernels was compared. A third-order polynomial kernel was found to be preferable to the other tested kernels based on the observation that the polynomial kernel provided satisfactory results for a smaller misclassification cost value. The results were compared across all the motor tasks. Although differences were observed among estimation error values for different motor tasks, several motor tasks performed equally well. This suggests that the proposed accelerometer features capture aspects of the movement patterns that are not specific to a given motor task. This further suggests that the proposed analyses could be extended to other motor tasks, possibly including recordings of activities of daily living.

Furthermore, we studied the impact on the estimation error of utilizing different combinations of the feature types. The primary objective of this part of the study was to assess whether reliable results could be achieved without relying on feature types that are associated with high power consumption requirements. Results indicated that it is possible to reliably estimate clinical scores on the basis of three feature types that are compatible with implementation on the SHIMMER platform: the rms value, the data range value, and two frequency-based features (i.e., the dominant frequency and the ratio of energy of the dominant frequency component to the total energy). When we utilized these features, we achieved average estimation error values of $3.4 \%$ for tremor, $2.2 \%$ for bradykinesia, and $3.2 \%$ for dyskinesia.

To our knowledge, the work presented in this paper is the first attempt of pursuing an integrated development of a wearable system and algorithms to assess the severity of symptoms and motor complications in patients with Parkinson's disease. The development of signal processing and pattern recognition algorithms was performed considering the power consumption requirements of an available body sensor network, i.e., the SHIMMER platform by Intel. The memory capacity of the SHIMMER platform allows raw accelerometer data to be stored on the nodes thus minimizing the amount of information to be transmitted through the radio, resulting in a significant decrease in power consumption. The implemented firmware used to estimate accelerometer data features on the nodes enables the option of transmitting features, as opposed to raw accelerometer data, when a spot check to assess the quality of the recordings is required. Therefore, the use of the radio in the proposed body sensor network configuration is limited to 1) sporadic bursts of information to synchronize the clocks of the different nodes and 2) periodic access to either the raw data or accelerometer feature sets to check the quality of the recordings during patient monitoring. This configuration allows us to monitor patients for several days without recharging the batteries of the body sensor network nodes.

Ongoing studies by our research team are focused on further developing the procedures presented in this paper. In the experiments performed in this study, we chose to ask patients to withdraw their medications prior to the data collection in order to maximize the observed magnitude of the changes in severity of symptoms and motor complications. Although this procedure is utilized in clinical settings, it would be preferable to monitor patients during "normal" motor fluctuation cycles. We have therefore initiated a study in which patients are observed without asking them to withdraw their medications. Another aspect of the study that we plan to expand upon is the number of symptoms and motor complications that are tracked during the monitoring period. For clinical purposes, it would be desirable to capture the severity of rigidity as well as the occurrence of freezing episodes (i.e., times when patients experience severe difficulties initiating movement). Finally, we plan to test the techniques presented in this paper in patients undergoing medication adjustments. Monitoring outcomes in this context would facilitate the titration of medications and would provide an objective (and easy to gather) measure of longitudinal changes in patient status that occur because of the degenerative nature of Parkinson's disease.

\section{ACKNOWLEDGMENT}

The authors would like to express their gratitude to F. Bomba, B. Kuris, S. Ayer, J. Healey, and the members of the Intel Digital Health Cambridge Group for their collaboration. Also, they would like to thank T. Hester, T. Lie-Nemeth, S. Salles, and D. Sherrill for their help in performing preliminary work that led to this paper. 


\section{REFERENCES}

[1] D. G. Standaert and A. B. Young, "Treatment of CNS neurodegenerative diseases," in Goodman and Gilman's Pharmacological Basis of Therapeutics, J. G. Hardman and L. E. Limbird, Eds. New York: McGraw-Hill, 2001, pp. 549-620.

[2] J. A. Obeso, C. W. Olanow, and J. G. Nutt, "Levodopa motor complications in Parkinson's disease," Trends Neurosci., vol. 23, pp. S2-S7, 2000.

[3] A. E. Lang and A. M. Lozano, "Parkinson's disease. First of two parts," N. Engl. J. Med., vol. 339, pp. 1044-1053, 1998.

[4] A. E. Lang and A. M. Lozano, "Parkinson's disease. Second of two parts," N. Engl. J. Med., vol. 339, pp. 1130-1143, 1998.

[5] W. J. Weiner, "Motor fluctuations in Parkinson's disease," Rev. Neurol. Dis., vol. 3, pp. 101-108, 2006.

[6] T. Muller and H. Russ, "Levodopa, motor fluctuations and dyskinesia in Parkinson's disease," Expert Opin. Pharmacother., vol. 7, pp. 1715-1730, 2006.

[7] P. S. Group, "Evaluation of dyskinesias in a pilot, randomized, placebocontrolled trial of remacemide in advanced Parkinson disease," Arch. Neurol., vol. 58, pp. 1660-1668, 2001.

[8] J. Ghika, A. W. Wiegner, J. J. Fang, L. Davies, R. R. Young, and J. H. Growdon, "Portable system for quantifying motor abnormalities in Parkinson's disease," IEEE Trans. Biomed. Eng., vol. 40, no. 3, pp. 276283, Mar. 1993.

[9] S. Spieker, C. Jentgens, A. Boose, and J. Dichgans, "Reliability, specificity and sensitivity of long-term tremor recordings," Electroencephalogr. Clin. Neurophysiol., vol. 97, pp. 326-331, 1995.

[10] P. Bonato, D. M. Sherrill, D. G. Standaert, S. S. Salles, and M. Akay, "Data mining techniques to detect motor fluctuations in Parkinson's disease," in Proc. Conf. IEEE Eng. Med. Biol. Soc., 2004, vol. 7, pp. 47664769.

[11] S. Patel, D. M. Sherrill, R. Hughes, T. Hester, N. Huggins, T. Lie-Nemeth, D. Standaert, and P. Bonato, "Analysis of the severity of dyskinesia in patients with Parkinson's disease via wearable sensors," presented at BSN2006-Int. Workshop Wearable Implantable Body Sens. Netw., Cambridge, MA, 2006.

[12] N. L. Keijsers, M. W. Horstink, J. J. van Hilten, J. I. Hoff, and C. C. Gielen, "Detection and assessment of the severity of levodopa-induced dyskinesia in patients with Parkinson's disease by neural networks," Mov. Disord., vol. 15, pp. 1104-1111, 2000.

[13] J. I. Hoff, A. A. van den Plas, E. A. Wagemans, and J. J. van Hilten, "Accelerometric assessment of levodopa-induced dyskinesias in Parkinson's disease," Mov. Disord., vol. 16, pp. 58-61, 2001.

[14] N. L. Keijsers, M. W. Horstink, and S. C. Gielen, "Automatic assessment of levodopa-induced dyskinesias in daily life by neural networks," Mov. Disord., vol. 18, pp. 70-80, 2003.

[15] N. L. Keijsers, M. W. Horstink, and S. C. Gielen, "Ambulatory motor assessment in Parkinson's disease," Mov. Disord., vol. 21, pp. 34-44, 2006.

[16] T. Thielgen, F. Foerster, G. Fuchs, A. Hornig, and J. Fahrenberg, "Tremor in Parkinson's disease: 24-hr monitoring with calibrated accelerometry," Electromyogr. Clin. Neurophysiol., vol. 44, pp. 137-146, 2004.

[17] P. Bonato, "Advances in wearable technology and applications in physical medicine and rehabilitation," J. Neuroeng. Rehabil., vol. 2, no. 1, article 2, 2005.

[18] E. Jovanov, A. Milenkovic, C. Otto, and P. C. de Groen, "A wireless body area network of intelligent motion sensors for computer assisted physical rehabilitation," J. Neuroeng. Rehabil., vol. 2, no. 1, article 6, 2005.

[19] T. Gao, D. Greenspan, M. Welsh, R. Juang, and A. Alm, "Vital signs monitoring and patient tracking over a wireless network," in Proc. Conf. IEEE Eng. Med. Biol. Soc., 2005, vol. 1, pp. 102-105.

[20] R. L. Elton, “Unified Parkinson's Disease Rating Scale," in Recent Developments in Parkinson's Disease, S. Fahn, Ed. New York: Macmillan, 1987, pp. 153-163.

[21] B. Post, M. P. Merkus, R. M. de Bie, R. J. de Haan, and J. D. Speelman, "Unified Parkinson's Disease Rating Scale motor examination: Are ratings of nurses, residents in neurology, and movement disorders specialists interchangeable?," Mov. Disord., vol. 20, pp. 1577-1584, 2005.

[22] R. Moddemeijer, "On estimation of entropy and mutual information of continuous distributions," Signal Process., vol. 16, pp. 233-246, 1989.

[23] V. Vapnik, The Nature of Statistical Learning Theory. New York: Springer-Verlag, 1995.
[24] R. P. W. Duin, P. Juszczak, P. Paclik, E. Pekalska, D. de Ridder, and D. M. J. Tax, PRTools4, A MATLAB Toolbox for Pattern Recognition. Delft, The Netherlands: Delft University of Technology, 2004.

[25] SHIMMER. [Online]. Available: http://docs.tinyos.net/index.php/ SHIMMER

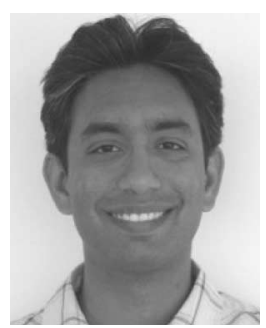

Shyamal Patel received the M.S. degree in electrical engineering from Rutgers University, New Brunswick, NJ, in 2005, and is currently working toward the $\mathrm{Ph} . \mathrm{D}$. degree in electrical engineering at Northeastern University, Boston, MA.

$\mathrm{He}$ is a Research Engineer in the Motion Analysis Laboratory, Spaulding Rehabilitation Hospital, Boston. His current research interests include the application of wearable technology for health monitoring by using advanced signal processing and pattern recognition techniques.

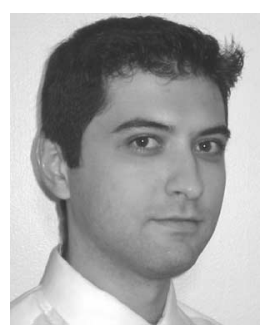

Konrad Lorincz received the M.S. degree in computer science from Harvard University, Cambridge, MA, where he is currently working toward the Ph.D. degree in computer science.

His current research interests include distributed systems, networks, wireless sensor networks, location tracking, and software engineering.

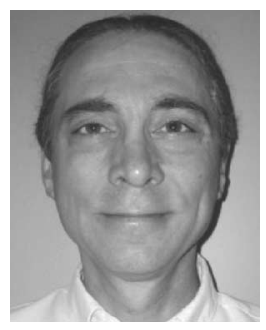

Richard Hughes received the M.S. degree from the Massachusetts General Hospital Institute of Health Profession, Boston.

He is currently a Physical Therapist with Partners Homecare, Beverly, MA. He is a Board-Certified Neurological Clinical Specialist and has served on the Spaulding Rehabilitation Hospital Internal Review Board. He has coauthored 14 papers and conference presentations. His current research interests include motor control and motor learning, clinical testing, and rehabilitation technology.

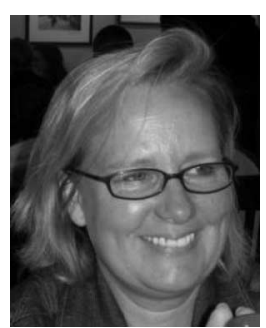

Nancy Huggins is a former nurse practitioner of Partners Parkinson Center. She developed a role for the nurse practitioner in the Parkinson Clinic and promoted outreach support, education, and exercise for people who live with Parkinson's disease with projects that partnered Massachusetts General Hospital in Boston and the National Parkinsons Foundation.

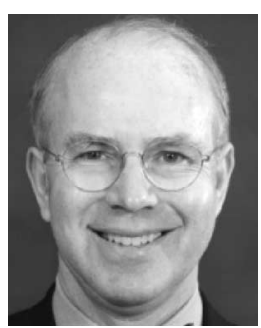

John Growdon received the M.D. degree from the University of Pennsylvania School of Medicine, Philadelphia.

He is currently the Director of the Massachusetts General Hospital (MGH) Movement Disorders Unit, Boston, which specializes in the diagnosis and care of Parkinson's disease. He is an Attending Neurologist at MGH and a Professor of Neurology at the Harvard Medical School, Boston. 


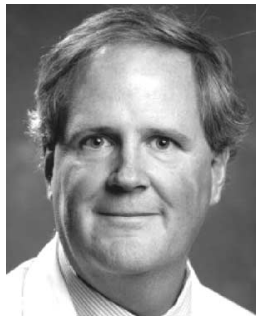

David Standaert received the M.D. and Ph.D. degrees from Washington University, St. Louis, MO.

He completed a one-year Internship in Medicine at Jewish Hospital of St. Louis followed by a three-year Neurology Residency at the University of Pennsylvania. He was appointed a Howard Hughes Medical Institute Physician Research Fellow, and completed a three-year Research and Clinical Fellowship in neurology (movement disorders) at Harvard Medical School and Massachusetts General Hospital in 1995. He subsequently joined the faculty at MGH, where he served as Director of the MGH/Massachusetts Institute of Technology Udall Center of Excellence in Parkinson's Disease Research. In July 2006, he joined the University of Alabama at Birmingham (UAB), Birmingham, and is the John and Juanelle Strain Professor of neurology. He serves as the Director of the Division of Movement Disorders, the Director of the American Parkinson Disease Association Advanced Center for Parkinson Research at UAB, and is the Director of the Center for Neurodegeneration and Experimental Therapeutics. His research is focused on understanding both the root causes of Parkinson's disease as well as the origin of the disabling symptoms that appear after longterm treatment of the disease.

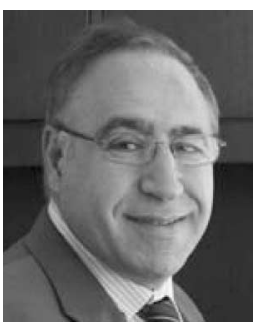

Metin Akay (S'87-M'91-SM'93-F'09) received the B.S. and M.S. degrees in electrical engineering from Bogazici University, Istanbul, Turkey, in 1981 and 1984, respectively, and the Ph.D. degree from Rutgers University, New Brunswick, NJ, in 1990.

$\mathrm{He}$ is currently a Professor of bioengineering at the School of Biological and Health Systems Engineering, Arizona State University, Tempe. He has promoted biomedical education in the world by writing several books and editing the Biomedical Engineering Book Series (Wiley and IEEE Press).

Prof. Akay is a Fellow of the Institute of Physics, and serves on numerous editorial and advisory boards of several international journals. He was the recipient of the IEEE Engineering in Medicine and Biology Society (EMBS) Service Award, the IEEE Third Millennium Medal, and the IEEE EMBS Early Career Achievement Award in 1997, the Young Investigator Award of Sigma Xi Society, Northeast Region, in 1998 and 2000.

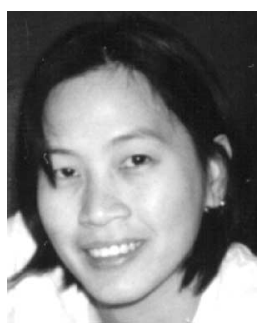

Jennifer Dy received the B.S. degree from the Department of Electrical Engineering, University of the Philippines, Quezon City, Philippines, in 1993, the M.S. and Ph.D. degrees from the School of Electrical and Computer Engineering, Purdue University, West Lafayette, IN, in 1997 and 2001.

She is an Associate Professor in the Department of Electrical and Computer Engineering, Northeastern University, Boston, MA, where she first joined the faculty in 2002. Her current research interests include machine learning, data mining, statistical pattern recognition, and their applications to biomedical analysis.

Dr. Dy was the Publications Chair for the International Conference on Machine Learning in 2004, and Program Committee Member for the International Conference on Machine Learning, the Association for Computing Machinery Special Interest Group on Knowledge Discovery and Data Mining, the Association for the Advancement of Artificial Intelligence, the International Joint Conference on Artificial Intelligence, and the SIAM International Conference on Data Mining. She is an Action Editor for the journal Machine Learning since 2007. She received a National Science Foundation Career Award in 2004.

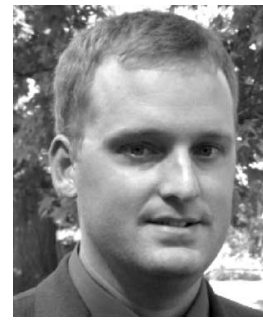

Matt Welsh (M'04) received the B.S. degree from Cornell University, Ithaca, NY, and the M.S. and $\mathrm{Ph}$.D. degrees from the University of California at Berkeley, Berkeley.

$\mathrm{He}$ is currently an Associate Professor of computer science at the Harvard School of Engineering and Applied Sciences, Cambridge, MA. His research interests involve operating system, network, and language support for complex distributed systems.

Dr. Welsh is a Senior Member of the Association for Computing Machinery and serves on the Editorial Board for ACM Transactions on Sensor Networks.

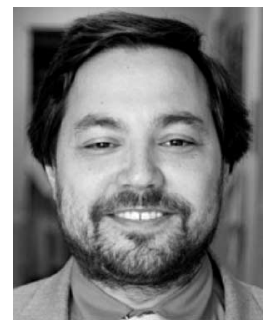

Paolo Bonato (S'93-M'95-SM'04) received the M.S. degree in electrical engineering from Politecnico di Torino, Turin, Italy and the Ph.D. degree from the Università di Roma "La Sapienza".

He serves as Director of the Motion Analysis Laboratory, Spaulding Rehabilitation Hospital, Boston, MA. He is an Assistant Professor in the Department of Physical Medicine and Rehabilitation, Harvard Medical School, Boston, and is a member of the Affiliated Faculty of the Harvard-MIT Division of Health Sciences and Technology, Cambridge, MA. His current research interests focus on technology in rehabilitation with special emphasis on wearable technology and robotics.

Dr. Bonato is an Elected Member of the IEEE Engineering in Medicine and Biology Society (EMBS) AdCom, and President of the International Society of Electrophysiology and Kinesiology. He served as Chair of the IEEE EMBS Technical Committee on Wearable Biomedical Sensors and Systems in 2008 and has been a member of this committee since its inception in 2006. 